\title{
Some chaotic properties of fuzzified dynamical systems
}

\author{
Cuina $\mathrm{Ma}^{{ }^{*}}$, Peiyong Zhu ${ }^{1}$ and Tianxiu $\mathrm{Lu}^{2}$
}

\author{
*Correspondence: \\ mcnmath@163.com \\ ${ }^{1}$ School of Mathematical \\ Sciences, University \\ of Electronic Science \\ and Technology of China, \\ 611731 Chengdu, People's \\ Republic of China \\ Full list of author information \\ is available at the end of the \\ article
}

\begin{abstract}
Let $X$ be a compact metric space and $f: X \longrightarrow X$ a continuous map. Considering the space $\mathbb{F}(X)$ of all nonempty fuzzy sets on $X$ endowed with the levelwise topology, we proved that its $g$-fuzzification is turbulent or erratic if the given system $f$ is turbulent or erratic correspondingly and $f$ is $\lambda$-expansive if and only if its $g$-fuzzification is $\lambda$-expansive.
\end{abstract}

Keywords: $g$-Fuzzification, Turbulent, Erratic, $\lambda$-Expansive

\section{Background}

Let $X$ be a compact metric space and $f$ be a continuous self-map. We denote by $\mathbb{F}(X)$ all nonempty fuzzy sets on $X$ endowed with the levelwise topology and we define $\widehat{f}$ as the Zadeh's extension of the map $f$ :

$$
\widehat{f}(u(x))=\sup _{y \in f^{-1}(x)}(u(y)), \quad u \in \mathbb{F}(X) .
$$

With the complexity of situations rising, the zadeh's extension has failed to be an accurate description for discrete fuzzy systems affected by the uncertainty. The time evolution of a chosen initial state is very important. We take the fuzzy set "short people" for example. "short people" in ancient time are not defined as short at present since the average height of people is increasing. It's not difficult to find some other examples to figure out that the zadeh's extension can't reflect the complex systems accurately. A very important generalization of the concept of zadeh's extension is $g$-fuzzification introduced in Kupka (2011a). It can be used for changing relevant membership grades and developed to describe the complex fuzzy systems in a more efficient way. The map $\widehat{f}_{g}: \mathbb{F}(X) \longrightarrow \mathbb{F}(X)$ is called $g$-fuzzification of $f$ and $\mathbb{F}(X)$ denotes all nonempty and fuzzy compact subsets of $X$. Over the last ten or so years, since many research works have been devoted to the chaotic behaviors of the fuzzified dynamical systems, such as, topological entropy, Devaney chaos, Li-Yorke chaos and its connections with erratic functions which has been done by Cánovas and Kupka (2011), Kupka (2011b), Diamond (1994), Diamond and Kloeden (1994), Diamond and Pokrovskii (1994). Recently, J.Kupka have studied various chaotic behaviors (Li-Yorke chaos, $\omega$-chaos, distributional chaos, topological chaos etc.) between a given dynamical system $(X, f)$ and its $g$-fuzzification.

(c) 2016 The Author(s) This article is distributed under the terms of the Creative Commons Attribution 4.0 International License (http://creativecommons.org/licenses/by/4.0/), which permits unrestricted use, distribution, and reproduction in any medium, provided you give appropriate credit to the original author(s) and the source, provide a link to the Creative Commons license, and indicate if changes were made. 
See more details in Kupka (2014). But it remains to be asked to study the other chaotic properties on $g$-fuzzification. Then the turbulence and erratic properties between a crisp dynamical system and its $g$-fuzzification are taken into our considerations in this paper.

On the other hand, the $\lambda$-expansive property in fuzzy systems is explored in this paper. Thus, the left work is to demonstrate the $\lambda$-expansiveness relationship between $\widehat{f}_{g}$ and $f$. Meanwhile, it is not difficult to see that the $\lambda$-expansive property can exhibit the sensitivity of $\widehat{f}_{g}$.

\section{Preliminaries}

\section{Metric space of fuzzy sets}

Let $(X, d)$ be a compact metric space and $C(X)$ denote all continuous functions. $f \in C(X)$ is a self-map. $\mathcal{K}(X)$ denotes all nonempty and compact subsets of $X$ with the Hausdorff metric $d_{H}$ defined by

$$
d_{H}(A, B)=\max \left\{\sup _{x \in A} \inf _{y \in B} d(x, y), \sup _{y \in B} \inf _{x \in A} d(x, y)\right\}
$$

for any $A, B \in \mathcal{K} . \bar{f}: \mathcal{K}(X) \longrightarrow \mathcal{K}$ is defined as $\bar{f}(A)=f(A)$ for any $A \in \mathcal{K}$.

A fuzzy set $u$ on the space $X$ is a function $u: X \longrightarrow I$ where $I=[0,1]$. For any $\alpha \in(0,1],[u]_{\alpha}=\{x \in X \mid u(x) \geq \alpha\}$ is called the $\alpha$-level of $u$ and $[u]_{0}=\{x \in X \mid u(x) \geq 0\}$ is the support of $u$ (shortly: supp(u)) (Román-Flores et al. 2011).

Throughout this paper, $\mathbb{F}(X)$ is defined as all upper semi-continuous fuzzy sets and equipped by the following metric:

$$
d_{\infty}(u, v)=\sup _{\alpha \in(0,1]} d_{H}\left([u]_{\alpha},[v]_{\alpha}\right) .
$$

Let

$$
\mathbb{F}^{\lambda}(X)=\{A \in \mathbb{F}(X) \mid A(x) \geq \lambda\}
$$

and $\mathbb{F}^{1}(X)$ is denoted as the system of all normal fuzzy sets on $\mathrm{X}$.

\section{$g$-fuzzifications}

Let $D_{m}(I)$ be the set of all nondecreasing right-continuous functions $g: I \longrightarrow I$, $I=[0,1]$. If $x=0$ and $x=1, g(x)=x$. A map $\widehat{f}_{g}$ denoted by

$$
\widehat{f}_{g}(x)=\sup _{y \in f^{-1}(x)}\{g(u(y))\} \text { for any } u \in \mathbb{F}(X), x \in X
$$

is defined as a $g$-fuzzification. Especially, it is the Zadeh's extension when the function $g(x)=1$. Also, a definition of $\alpha$-cut $[A]_{\alpha}^{g}$ is presented by $[A]_{\alpha}^{g}=\left\{x \in[A]_{0} \mid g(A(x)) \geq \alpha\right\}$. The following equation in Kupka (2011a) is indispensable.

$$
f\left([A]_{\alpha}^{g}\right)=\left[\widehat{f}_{g}(A)\right]_{\alpha}, \quad \forall A \in \mathbb{F}(X) .
$$


Lemma 1 Kupka (2014): Let $f \in C(X)$ and $g \in D_{m}(I)$. For any $\alpha \in(0,1]$ and nonempty fuzzy set $A \in \mathbb{F}(X)$, if $[A]_{\alpha}^{g} \neq \emptyset$ then there is $c \in(0,1]$ such that $[A]_{\alpha}^{g}=[A]_{c}$.

To explore some properties of $g$-fuzzification, for any $U \subset X$, we define

$$
e_{\alpha}(U)=\left\{\mathrm{B} \in \mathbb{F}(X) \mid[B]_{\alpha} \neq \emptyset \text { and }[B]_{\alpha} \subseteq U\right\}
$$

and

$$
\begin{aligned}
& \vartheta(U)=e_{1}(U) \cap e(U), \\
& e(U)=\{A \in \mathbb{F}(X) \mid \operatorname{supp}(A) \subseteq U\} .
\end{aligned}
$$

Clearly, $e(U) \neq \emptyset$ if and only if $U \neq \emptyset$. Moreover, the following lemma is essential in the paper.

Lemma 2 Kupka (2014): Let $U$, $V$ be two subsets of $X, f \in C(X)$ and $g \in D_{m}(I)$. Then:

1) $e(U \cap V)=e(U) \cap e(V)$.

2) $\widehat{f}_{g}(e(U)) \subseteq e(f(U))$.

3) $\widehat{f}_{g}(e(U))=e(f(U))$ whenever $U$ is closed.

Likewise, $\vartheta(U)$ has the same properties compared with $e(U)$.

Lemma 3 Kupka (2014): Let $U$, $V$ be two subsets of $X, f \in C(X)$ and $g \in D_{m}(I)$. Then:

1) $\vartheta(U \cap V)=\vartheta(U) \cap \vartheta(V)$.

2) $\widehat{f}_{g}(\vartheta(U)) \subseteq \vartheta(f(U))$.

3) $\widehat{f}_{g}(\vartheta(U))=\vartheta(f(U))$ whenever $U$ is closed.

Turbulence, erratic property, Block and Coppel chaos and $\lambda$-expansiveness

Before we present the elegant results, we need to introduce some basic definitions of chaotic behavior explored in this paper.

Definition 1 Román-Flores et al. (2011): Let $f: X \longrightarrow X$ be a continuous function. We say that $f$ is a turbulent function if there are disjoint nonempty closed subsets $J, K$ of $X$ such that:

$$
J \cup K \subseteq f(J) \cap f(K)
$$

Definition 2 Román-Flores et al. (2011): A map $f \in C(X)$ is erratic if there exists a nonempty closed set $A \subseteq X$ satisfying the following two conditions:

1) $A \cap f(A)=\emptyset$.

2) $A \cup f(A) \subseteq f^{2}(A)$.

Definition 3 Román-Flores et al. (2011): Let $f: X \longrightarrow X$ be a continuous function.We say that $f$ is chaotic in the sense of Block and Coppel (in short: B-C chaos) if and only if its iterates is turbulent, i.e., there exists $n \geq 1$ and two disjoint nonempty compact subsets $J, K$ of $X$ such that 
$J \cup K \subseteq f^{n}(J) \cap f^{n}(K)$

It should be remarkable that the erratic property is stronger than B-C chaos.

Definition 4 Let $f: X \longrightarrow X$ be a function. We claim that $f$ is expansive if and only if there exists a real constant $\lambda>1$ such that

$$
d(f(x), f(y)) \geqslant \lambda d(x, y), \forall x, y \in X .
$$

In this case we claim that $f$ is $\lambda$-expansive Román-Flores and Chalco-Cano (2005).

\section{Main results}

To prove the turbulent and erratic properties, firstly, a theorem on $e(U)$ is given here.

Theorem 1 Let $U$ be closed subset of $X$ and $e(U)=\{\mathrm{B} \in \mathbb{F}(X) \mid \operatorname{supp}(\mathrm{B}) \subseteq U\}, f \in C(X)$ and $g \in D_{m}(I)$. Then,

$$
\widehat{f_{g}^{n}}(e(U))=\widehat{f}_{g}^{n}(e(U)) .
$$

Proof It can be proved by the mathematical induction.

When $n=1$, Left $=$ Right $=\widehat{f}_{g}(e(U))$. Clearly, the theorem holds.

Assume that the theorem is true for $n=k$, i.e.,

$$
\widehat{f}_{g}^{k}(e(U))=\widehat{f}_{g}^{k}(e(U))
$$

By the Lemma 2 in Kupka (2014), we have

$$
\widehat{f_{g}^{k}}(e(U))=e\left(f^{k}(U)\right) .
$$

When $n=k+1$,

$$
\begin{aligned}
\widehat{f_{n}^{k+1}}(e(U)) & =e\left(f_{n}^{k+1}(U)\right)=e\left(f_{n} \circ\left(f_{n}^{k}(U)\right)\right) \\
& =\widehat{f}_{n}\left(e\left(f_{n}^{k}(U)\right)\right) \\
& =\widehat{f}_{n} \circ \widehat{f}_{n}^{k}(e(U)) \\
& =\widehat{f}_{n}^{k+1}(e(U)) .
\end{aligned}
$$

Consequently, the statement is proved completely.

Likewise, we can achieve the similar result on $\vartheta(U)$.

Theorem 2 Let $U$ be closed subset of $X$ and $\vartheta(U)=e_{1}(U) \cap e(U) . f \in C(X)$ and $g \in D_{m}(I)$. Then,

$$
\widehat{f_{g}^{n}}(\vartheta(U))=\widehat{f}_{g}^{n}(\vartheta(U)) .
$$


Here, we make a point that $\forall \alpha \in(0,1]$,

$$
\widehat{f_{g}^{n}}\left(e_{\alpha}(U)\right) \neq \widehat{f}_{g}^{n}\left(e_{\alpha}(U)\right) .
$$

See Lan and $\mathrm{Mu}$ (2014) for more details.

Román-Flores et al. (2011) explored the dynamics of $\widehat{f}$ and $f$. These conclusions can be listed as follows:

$$
\begin{aligned}
& f \text { is turbulent (erratic) } \Rightarrow \widehat{f} \text { is turbulent (erratic) } \\
& \widehat{f} \text { is turbulent (erratic) } \nRightarrow f \text { is turbulent (erratic). }
\end{aligned}
$$

More precisely, Román-Flores et al. (2011) present some examples in order to show the irreversible links between $f$ and $\widehat{f}$. We generalizes the results with $\widehat{f}_{g}$. As the Zadeh's extension is a spacial case of the g-fuzzification, we can make a conclusion:

$$
\widehat{f}_{g} \text { is turbulent (erratic) } \nRightarrow f \text { is turbulent (erratic). }
$$

The left work is to prove the following implication:

$$
f \text { is turbulent (erratic) } \Rightarrow \widehat{f}_{g} \text { is turbulent (erratic). }
$$

Theorem 3 If $f \in C(X), f: X \longrightarrow X$ is a turbulent function, then $\widehat{f}_{g}: \mathbb{F}(X) \longrightarrow \mathbb{F}(X)$ is a turbulent function.

Proof Since $f$ is turbulent, there exists nonempty and closed $U, V \subseteq X$ and $U \cap V=\emptyset$ such that $U \cup V \subseteq f(U) \cap f(V)$.

By the definition of $e(U)$, clearly, $e(U)$ and $e(V)$ are two disjoint nonempty closed subsets of $\mathbb{F}(X)$. Applying the Lemma 2, we have

$$
\begin{aligned}
e(U) \cup e(V) \subseteq e(U \cup V) & \subseteq e(f(U) \cap f(V)) \\
& =e(f(U)) \cap e(f(V)) \\
& =\widehat{f}_{g}(e(U)) \cap \widehat{f}_{g}(e(V)) .
\end{aligned}
$$

Finally, we can conclude that $\widehat{f}_{g}$ is a turbulent function.

Theorem 4 Let $f \in C(X)$ be erratic, then, $\widehat{f}_{g}$ is a erratic function.

Proof Since $f$ is erratic, there exists a nonempty closed subset $U \subseteq X$ such that $U \cap f(U)=\emptyset$ and $U \cup f(U) \subseteq f^{2}(U)$. Thus, $e(U)$ is nonempty closed subset of $\mathbb{F}(X)$ and

$$
\begin{aligned}
e(U) \cap \widehat{f}_{g}(e(U)) & =e(U) \cap e(f(U)) \\
& =e((U) \cap f(U)) \\
& =e(\emptyset)=\emptyset .
\end{aligned}
$$


On the other hand, using the Theorem 1 and the Lemma 2, we have

$$
\begin{aligned}
e(U) \cup \widehat{f}_{g}(e(U)) & =e(U) \cup e(f(U)) \\
& \subseteq e(U \cup f(U)) \\
& \subseteq e\left(f^{2}(U)\right) \\
& =\widehat{f}_{g}^{2}(e(U)) .
\end{aligned}
$$

Consequently, $\widehat{f}_{g}$ is erratic.

Corollary 1 Let $f \in C(X)$ be erratic, then, $\widehat{f}_{g}$ is a B-C chaos function.

Remark 1 Because $\vartheta(U)$ has similar properties to the $e(U)$, it can be verified with $\vartheta(U)$ that the statements are true. Comparing Theorem 3 with Theorem 4, the fuzzy set containing $e(U)$ at least is perfect for the two theorems to make sense.

Next, we shall discuss the $\lambda$-expansive property of $\widehat{f}_{g}$.

Theorem 5 Let $f: X \longrightarrow X$ be a continuous function and $g \in D_{m}(I)$. Then $f$ is $\lambda$ -expansive if and only if $\widehat{f}_{g}$ is $\lambda$-expansive.

Proof $(\Rightarrow)$ Since $f$ is $\lambda$-expansive, for any $u, v \in \mathbb{F}(X)$ and $\alpha \in(0,1]$, it follows that

$$
\begin{aligned}
\sup & d_{H}\left(f\left([u]_{\alpha}\right), f\left([v]_{\alpha}\right)\right) \\
= & \max _{\alpha \in(0,1]}\left\{\sup _{x \in[u]_{\alpha}} \inf _{y \in[v]_{\alpha}} d(f(x), f(y)),\right. \\
& \left.\sup _{y \in[v]_{\alpha}} \inf _{x \in[u]_{\alpha}} d(f(x), f(y))\right\} \\
\geq & \max _{\alpha \in(0,1]}\left\{\sup _{x \in[u]_{\alpha}} \inf _{y \in[v]_{\alpha}} \lambda d(x, y), \sup _{y \in[v]_{\alpha}} \inf _{x \in[u]_{\alpha}} \lambda d(x, y)\right\} \\
= & \lambda \max _{\alpha \in(0,1]^{\prime}}\left\{\sup _{x \in[u]_{\alpha}} \inf _{y \in[v]_{\alpha}} d(x, y), \sup _{y \in[v]_{\alpha}} \inf _{x \in[u]_{\alpha}} d(x, y)\right\} \\
= & \lambda \sup d_{H}\left([u]_{\alpha},[v]_{\alpha}\right) \\
= & \lambda d_{\infty}(u, v) .
\end{aligned}
$$

Applying $f\left([A]_{\alpha}^{g}\right)=\left[\widehat{f}_{g}(A)\right]_{\alpha}$, it follows that

$$
\begin{aligned}
d_{\infty}\left(\widehat{f}_{g}(u), \widehat{f}_{g}(v)\right) & =\sup d_{H}\left(\left[\widehat{f}_{g}(u)\right]_{\alpha},\left[\widehat{f}_{g}(v)\right]_{\alpha}\right) \\
& =\sup d_{H}\left(f\left([u]_{\alpha}^{g}\right), f\left([v]_{\alpha}^{g}\right)\right) .
\end{aligned}
$$

By the Lemma 1 , there is $c \in(0,1]$ such that

$$
\begin{aligned}
\sup d_{H}\left(f\left([u]_{\alpha}^{g}\right), f\left([v]_{\alpha}^{g}\right)\right) & =\sup d_{H}\left(f\left([u]_{c}\right), f\left([v]_{c}\right)\right) \\
& \geq \lambda d_{\infty}(u, v),
\end{aligned}
$$

which implies $\widehat{f}_{g}$ is $\lambda$-expansive.

$(\Leftarrow)$ Based on the two following equations:

$$
d_{\infty}\left(\chi_{\{x\}}, \chi_{\{y\}}\right)=d(x, y)
$$


and

$$
d_{\infty}\left(\widehat{f}_{g}\left(\chi_{\{x\}}\right), \widehat{f}_{g}\left(\chi_{\{y\}}\right)\right)=d(f(x), f(y))
$$

it is obvious that $f$ is $\lambda$-expansive.

Corollary 2 If $\widehat{f}_{g}$ is $\lambda$-expansive, $\lambda>1$, then $\widehat{f}_{g}$ is sensitively dependent.

Proof Applying the method of induction, it is obvious that

$$
d_{\infty}\left(\widehat{f}_{g}^{n}(u), \widehat{f}_{g}^{n}(v)\right) \geq \lambda^{n} d_{\infty}(u, v) \rightarrow \infty \text {, as } n \rightarrow \infty
$$

Thus, $\widehat{f}_{g}$ is sensitively dependent.

Corollary 3 Iff is $\lambda$-expansive, then $\widehat{f}_{g}$ is sensitively dependent.

\section{Conclusions}

In this paper, exploiting the turbulent and erratic properties, we develop the ideas of Román-Flores et al. (2011) and present some properties of $e(U)$ and $\vartheta(U)$ for $\widehat{f}_{g}$, which can be applied to the proof of Theorem 3 and Theorem 4. Moreover, the $\lambda$-expansive property between $f$ and $\widehat{f}_{g}$ is studied and exhibit the sensitivity. Inducing sensitivity on fuzzy systems contains asymptotic sensitive, Li-Yorke sensitive and spatial-temporal sensitive etc, which will be further investigated and solved in a later work.

\section{Authors' contributions}

This work was carried out in collaboration among the authors. CM, PZ and TL have a good contribution to design the study, and to perform the analysis of this research work. All authors read and approved the final manuscript.

\section{Author details}

1 School of Mathematical Sciences, University of Electronic Science and Technology of China, 611731 Chengdu, People's Republic of China. ${ }^{2}$ Department of Mathematics, Sichuan University of Science and Engineering, 643000 Zigong, People's Republic of China.

\section{Acknowledgements}

This work was supported by National Natural Science Foundation of China (11501391) and the Scientific Research Project of Sichuan University of Science and Engineering (2014RC02).

Competing interests

The authors declare that they have no competing interests.

Received: 20 January 2016 Accepted: 6 May 2016

Published online: 17 May 2016

\section{References}

Cánovas JS, Kupka J (2011) Topological entropy of fuzzified dynamical systems. Fuzzy Sets Syst 165(1):37-49

Diamond P (1994) Chaos in iterated fuzzy systems. J Math Anal Appl 184(3):472-484

Diamond P, Kloeden PE (1994) Metric spaces of fuzzy sets: theory and applications. World scientific, Singapore

Diamond P, Pokrovskii A (1994) Chaos, entropy and a generalized extension principle. Fuzzy Sets Syst 61(3):277-283

Kupka J (2011a) On fuzzifications of discrete dynamical systems. Inf Sci 181(13):2858-2872

Kupka J (2011 b) On devaney chaotic induced fuzzy and set-valued dynamical systems. Fuzzy Sets Syst 177(1):34-44

Kupka J (2014) Some chaotic and mixing properties of fuzzified dynamical systems. Inf Sci 279:642-653

Lan Y, Mu C (2014) Martelli chaotic properties of a generalized form of Zadeh's extension principle. J Appl Math 2014. doi:10.1155/2014/956467

Román-Flores H, Chalco-Cano Y, Silva GN, Kupka J (2011) On turbulent, erratic and other dynamical properties of Zadeh's extensions. Chaos Solitons Fractals 44(11):990-994

Román-Flores H, Chalco-Cano Y (2005) Robinson's chaos in set-valued discrete systems. Chaos Solitons Fractals 25(1):33-42 